\title{
La capacidad competitiva de los profesionales desarrolladores de tecnología en Tegucigalpa: una métrica para evaluar la equidad salarial en el mercado laboral hondureño
}

\author{
Jesús David Argueta Moreno \\ Crisley Dávila Laínez ${ }^{2}$
}

\section{RESUMEN}

En el campo de la administración y de forma particular en la gestión del recurso humano, el estudio de los activos intangibles ha generado un gran interés en el ámbito empresarial, pues de su análisis se desprenden las potencialidades competitivas de los profesionales en el ámbito laboral, el cual bajo la premisa de fortalecer el capital intelectual de sus asociados procura la obtención de un mejor desempeño de estos.

Por lo anterior, con esta investigación se pretende la valoración monetizada del capital intelectual en el marco del sector desarrollador de software de Tegucigalpa, Francisco Morazán, con el objetivo determinar si los profesionales en la rama de la ingeniería que laboran en el rubro previamente mencionado, devengan un salario mensual congruente con su capital intelectual $(\mathrm{Cl})$.

Cabe mencionar que la investigación se efectuó en 23 empresas del sector desarrollador de software radicadas en la ciudad de Tegucigalpa, Francisco Morazán, Honduras. La metodología usada fue el enfoque mixto y diseño no experimental, cuyos resultados indican que los profesionales que laboran en esta rama se encuentran subvalorados en alrededor de Lps. 22,000.00; cifra que denota profundas inconsistencias en el mercado laboral local.

Palabras clave: equidad, capital intelectual, grado académico, competencias y experiencia.

\footnotetext{
${ }^{1}$ Profesor universitario de la Facultad de Ciencias Económicas, UNAH: jargueta@iies-unah.org

${ }^{2}$ Profesor universitario de la Facultad de Relaciones Internacionales, Universidad Católica de Honduras: davilac.lainez@gmail.com
} 


\section{ABSTRACT}

Along the fields of administration and particularly the human resource management, highlights of great importance are growing around the study of intangible assets, on the pursuit of a quantitative overall analysis on the competitive environment, that entails the professional's performance on their workplace, through the quantification of the Intellectual Capital.

Therefore, this research seeks the monetized valuation of the Intellectual Capital, under the software developer sector, allocated in the city of Tegucigalpa Francisco Morazán, in order to determine if the professionals in the branch of engineering that work in the category previously mentioned, earn a congruent monthly salary in relation to their Intellectual Capital (IC). Despite the use of a mixed approach (Cualitative and Cuantitative Scope) towards this study, who obeyed a non experimiental design, the results obtained unveil that the professionals previously mentioned, earn a salary who underestimates their current competences in around 22,000 lempiras.

It should be mentioned that the research was conducted on 23 software developing companies allocated the city of Tegucigalpa Francisco Morazán, Honduras.

Key words: equity, intellectual capital, academic degree, competence, experience. 


\section{INTRODUCCIÓN}

La crisis mundial tiene la particularidad de exigir nuevas y más demandantes competencias, pericias y habilidades, al forzar a los nuevos profesionales a adquirir destrezas y argumentos cognitivos más competitivos para así destacarse entre los más connotados candidatos a contratar en mercados laborales saturados.

La búsqueda de nuevos espacios que faciliten la inserción de estos profesionales en el mercado laboral local o internacional describe una movilización demográfica interesante, manifiesta en el éxodo de jóvenes talentos al exterior (Argueta, 2013).

Una vez insertados en el ámbito laboral, es responsabilidad de estos profesionales el procurar que las tasas salariales devengadas sean proporcionales a su grado académico, experiencia y competencias (primarias y secundarias) adquiridas entorno a un crecimiento profesional sostenible (Bondi, 2001). La gestión que involucra el análisis de estas habilidades, competencias, conocimientos y pericias, comprende la definición del concepto tipificado como capital intelectual (Campos, 1999). Sin embargo, ante la necesidad de atraer y mantener a los profesionales talentosos ya reclutados, las empresas han ideado distintas métricas y herramientas para valorar con mayor precisión y menor subjetividad el capital intelectual de sus asociados y acorde a estos insumos definir las tasas salariales pertinentes (Becker, 1964).

En este contexto surge la iniciativa de adaptar un instrumento de cuantificación del $\mathrm{Cl}$, con la finalidad de mitigar la posible sub o sobrevaloración del personal en el ámbito empresarial, dando cabida a la presente investigación. En consecuencia, el actual documento tiene como fin la descripción integral de las entradas, herramientas, metodología, procesos y salidas/resultados abordados por el proyecto de investigación denominado: La capacidad competitiva de los profesionales desarrolladores de tecnología en Tegucigalpa: una métrica para evaluar la equidad salarial en el mercado laboral hondureño.

\section{METODOLOGÍA}

La presente investigación de corte cualitativo-cuantitativo, gira entorno a la evaluación y cuantificación de las potencialidades cognitivas del profesional en la rama de la ingeniería que labora en el sector desarrollador de software de 
Tegucigalpa, Francisco Morazán, el cual devenga un salario mensual que podría no ser congruente con el valor monetizado de su capital intelectual.

El estudio se realizó en 23 empresas pertenecientes al sector desarrollador de productos tecnológicos (software), con ubicación en la ciudad de Tegucigalpa, Francisco Morazán, entrevistando a 96 profesionales de la rama de la ingeniería que laboran en estas instituciones, los cuales se dedican al desarrollo de software.

\section{DEFINICIÓN DEL PROBLEMA}

En la actualidad, el sector desarrollador de tecnología en Honduras cuenta con más de 150 empresas en el país, las cuales afrontan un alarmante decremento en sus ventas brutas anuales promedio por empresa en alrededor de USD 19,000.00; representando un claro peligro entorno al deterioro de la capacidad financiera del sector, el cual de forma directa e indirecta deberá de sobrellevar los siguientes impactos:

1. Una menor liquidez entre las empresas desarrolladoras de software.

2. Beneficios inferiores para los accionistas.

3. Una menor capacidad de reclutamiento/absorción del recurso humano.

4. Una insuficiente capacidad de reacondicionar las tasas salariales del personal vigente a propuestas más atractivas o niveladas a las necesidades del mercado regional.

5. Otros: "Recordando que las empresas desarrolladoras de tecnología dependen en gran medida del talento de cada uno de sus operarios" (Giarelli, 2013).

Por lo anterior, de forma puntual y abierta se manifiesta que para contrarrestar la actual crisis salarial y así resguardar los beneficios del profesional que cuenta con un $\mathrm{Cl}$ alto, ya que ante la inexistencia de un perfil ideal a nivel de la academia (ofertantes de profesionales) versus la inexistencia de un perfil idóneo a nivel del empresariado (demandantes de profesionales), este análisis determinó los puntos de intersección de ambos perfiles, para adoptar una herramienta capaz de evaluar y cuantificar por sí misma con la suficiente precisión y nivel de confianza del capital intelectual de estos profesionales (Giarelli, 2013). 
Ante este vacío financiero y del conocimiento, el Estado de Honduras procuró una nivelación general de la tasa salarial en el año 2012, al fijar un nuevo ajuste salarial en el país, en donde los sueldos ascendieron de 4,600 lempiras (mensuales) en su categoría más baja representada por el sector agrícola, a 7,140 lempiras (mensuales) en el área financiera; lo que generó un salario promedio de 5,870 lempiras (USD 309); según los datos proporcionados por la Secretaría de Trabajo y Seguridad Social (STSS) en ese año, Honduras se convirtió en el tercer país de Centroamérica con el salario mínimo más alto (USD 309), después de Panamá (USD 450) y Costa Rica (USD 375).

Sin embargo, este esfuerzo no produjo un impacto positivo en todos los rubros o sectores del país, destacando entre ellos que en la industria desarrolladora de software se generó un clima de inestabilidad laboral ante la incapacidad de estas empresas de lidiar con las nuevas exigencias salariales de sus asociados.

Asimismo, tras evaluar el análisis competitivo a nivel de país del World Economic Forum para el año 2013, al examinar 2 de sus 12 pilares fundamentales (5 y 7) se aprecia que tanto la educación superior hondureña como el mercado laboral local, cuentan con bajos niveles de eficiencia y eficacia, lo que supone una desmesurada compensación salarial para algunos sectores que más allá de contar con una mano de obra poco calificada, las restricciones particulares del sector impiden que este albergue a más profesionales o compense de mejor manera a los que actualmente laboran en él, en este caso el sector desarrollador de software de Tegucigalpa.

Por lo anterior, se pretende que esta investigación adapte una herramienta que se ajuste a las necesidades del sector y catalice el sustento teórico y metodológico necesario para agilizar la toma de decisiones de los directivos y gestores del recurso humano, respecto a la apropiada remuneración de sus asociados, en consonancia con las características profesionales y académicas que esté presente en el contexto local.

\section{HIPÓTESIS}

1. La variable capital intelectual incide sobre la variable tasa salarial al presentar una relación directa con los montos salariales mensualmente devengados por los profesionales de la ingeniería que laboran en las empresas desarrolladoras de software en la ciudad de Tegucigalpa, Honduras. 
2. La cuantificación de los profesionales en la rama de la ingeniería que laboran en el sector desarrollador de software demostró que su capital intelectual monetizado es inferior (<) a su remuneración actual de mercado, lo que sugiere una sobrevaloración del profesional en este sector.

Descripción de las variables

Variable capital intelectual (Cl)

¿Qué es capital intelectual? Según el economista Eduardo Bueno Campos, el término capital intelectual representa la valorización de los activos intangibles creados por los flujos de conocimiento de la empresa o el conjunto de competencias básicas distintivas (personales, organizativas, tecnológicas y relacionales) de carácter intangible que permiten crear y sostener la ventaja competitiva (Pfeffer, 1994). Estos flujos del conocimiento evolucionan a través de 3 etapas, representadas en la figura 1.

Figura 1. Proceso de la creación de las sociedades del conocimiento
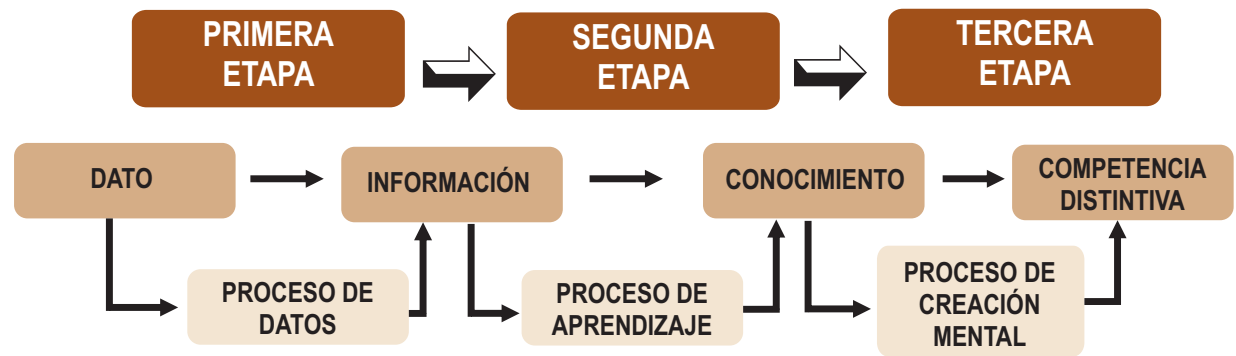

Fuente: Campos, 1999.

De igual forma, en la figura 1 se describe la evolución del conocimiento a través de la transición entre la captura del sustento teórico/dato y su recorrido en 7 procesos hasta la obtención de las nuevas y distintivas competencias. Aquí el conocimiento es el principal insumo de este sistema, el cual se canaliza de forma aislada y se articula en pequeños bloques denominados procesos, a los cuales se les articula y da sentido, teniendo como resultado estructuras informativas, las cuales se aglutinan en unidades de aprendizaje, capitalizadas por el asociado como conocimiento. Finalmente, al llenar un vacío puntual del conocimiento individual se desarrolla una gestión cognitiva que despliega nuevas competencias manifiestas en el empleado/organización (Campos, 2003). 
Dimensiones de la variable capital intelectual

Al articular la investigación de forma directa con el modelo de dirección por competencias propuesto por Eduardo Bueno en 1998 (Zacapa, 2005), se procura la descripción de la variable capital intelectual en el marco de la siguiente tipología:

Capital intelectual $(\mathrm{Cl})=$ capital humano $(\mathrm{CH})+$ capital tecnológico $(\mathrm{CT})+$ capital relacional $(\mathrm{CR})+$ capital organizacional $(\mathrm{CO})$ :

- Capital humano

- Capital tecnológico

- Capital relacional

- Capital estructural

Para cada una de las dimensiones del capital intelectual se diseminó a través de 3 componentes:

- $\operatorname{Actitudes}(\mathrm{A})$

- Conocimientos(Co)

- Capacidades (Ca)

Estos componentes, a su vez, se articulan de forma directa con la aplicación latente y manifiesta de 4 tipos de competencias:

- $\mathrm{h}=$ Competencias expresas

- $0=$ Competencias expresas de la organización

- $\mathrm{t}=$ Competencias tecnológicas

- $r=$ Competencias relacionadas

Por tanto, cada dimensión del capital intelectual se podrá cuantificar al estudiar la articulación de los siguientes componentes y competencias descritos en la tabla 1. 
Tabla 1. Estructuración del dimensionamiento del capital intelectual

\begin{tabular}{|l|l|l|}
\hline \multicolumn{1}{|c|}{ Dimensiones } & \multicolumn{1}{c|}{ Componentes } & \multicolumn{1}{c|}{ Competencias } \\
\hline Capital humano & $\begin{array}{l}\text { Actitudes } \\
\text { Conocimientos } \\
\text { Capacidades }\end{array}$ & $\begin{array}{l}\text { Competencias } \\
\text { expresas }\end{array}$ \\
\hline Capital tecnológico & $\begin{array}{l}\text { Actitudes } \\
\text { Conocimientos } \\
\text { Capacidades }\end{array}$ & $\begin{array}{l}\text { Competencias } \\
\text { tecnológicas }\end{array}$ \\
\hline Capital relacional & $\begin{array}{l}\text { Actitudes } \\
\text { Conocimientos } \\
\text { Capacidades }\end{array}$ & $\begin{array}{l}\text { Competencias } \\
\text { relacionadas }\end{array}$ \\
\hline Capital estructural & $\begin{array}{l}\text { Actitudes } \\
\text { Conocimientos } \\
\text { Capacidades }\end{array}$ & $\begin{array}{l}\text { Competencias } \\
\text { expresas de la } \\
\text { organización }\end{array}$ \\
\hline
\end{tabular}

Fuente: elaboración propia basada.

De igual forma, al analizar el componente denominado competencias expresas de la organización, abordado en la estructura de la tabla 2, se destaca su división.

Tabla 2. Competencias básicas expresas de la organización

Competencias básicas que componen al capital humano
Nivel de competencias de los trabajadores
Satisfacción del personal
Capacidad de innovación
Formación académica
Competencias básicas que componen al capital relacional
Satisfacción del cliente
Lealtad del cliente
Notoriedad de la marca
Calidad del servicio
Competencias básicas que componen al capital organizacional
Trabajo en equipo
Competencias básicas que componen al capital tecnológico
Tecnología de la información
Propiedad intelectual
Investigación y desarrollo
Fuente: Zacapa, 2005 .

Sin embargo, para efectos de la tropicalización del instrumento será necesaria la adaptación de las competencias específicas del sector en el marco del rubro desarrollador de software en Tegucigalpa, Francisco Morazán (ver figura 2). Tras describir las dimensiones, componentes y subcomponentes del capital intelectual (Campos, 2003), es válido citar que este se compone de la suma de sus 4 
dimensiones, las cuales se diseminan en distintos valores, competencias y conocimientos particulares, dando como resultado, la siguiente sumatoria:

Fórmula de la cuantificación del capital intelectual:

$$
\begin{gathered}
\mathrm{CI}=[\mathrm{Ah}+\mathrm{Coh}+\mathrm{Cah}]+[\mathrm{Ao}+\mathrm{Coo}+\mathrm{Cao}]+[\mathrm{At}+\mathrm{Cot}+\mathrm{Cat}]+ \\
{[\mathrm{Ar}+\mathrm{Cor}+\mathrm{Car}]}
\end{gathered}
$$

Fuente: Campos, 2003.

En consecuencia, es vital recordar que esta investigación utilizará como su modelo de línea base a la propuesta de Eduardo Bueno denominada "Modelo de dirección por competencias", la cual pretende cuantificar el capital intelectual a través de sus 4 dimensiones (capital tecnológico, capital humano, capital relacional y capital estructural) y las distintas competencias inherentes en ellas, efectuado por Teonila García Zacapa, descrito en la figura 2 (Zacapa, 2005).

Figura 2. Modelo de Eduardo Bueno interpretado por Teonila García Zacapa

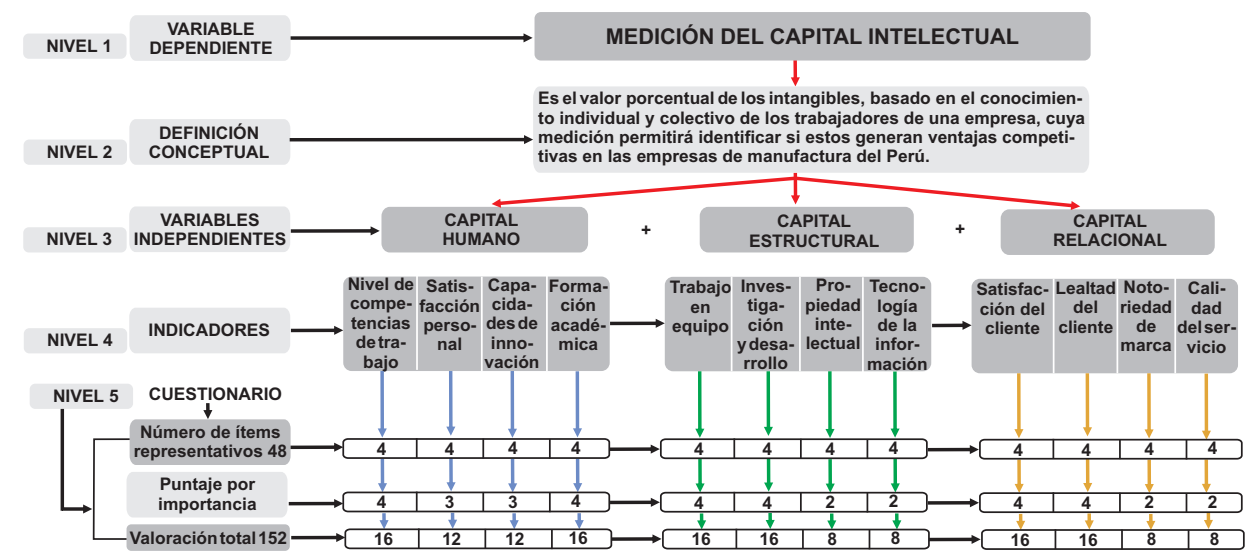

Fuente: Zacapa, 2007.

Cabe destacar que la creación de los instrumentos de este estudio se efectuaron en el marco de esta reflexión, con la finalidad de adaptar esta percepción sobre las competencias de los profesionales de la rama de ingeniería que laboran en el sector desarrollador de tecnología en la ciudad de Tegucigalpa.

Perfil de las empresas desarrolladoras de software en Honduras

Tras evaluar la cantidad de 23 empresas pertenecientes al sector desarrollador de tecnología (software) en la ciudad de Tegucigalpa, se destacan las características 
generales identificadas a la luz de los datos recados en el instrumento de medición y las visitas efectuadas a cada una de ellas, sin embargo, se efectuará el contraste con los insumos previamente tipificados en el informe recabado a nivel nacional publicado por la Asociación Hondureña de Tecnología de Información (AHTI) en noviembre de 2013.

Acorde con las empresas desarrolladoras de productos tecnológicos evaluadas en el estudio (empresas desarrolladoras de software), se infiere que estas gestionan un promedio de 4 profesionales en la rama de la ingeniería, no obstante, la AHTI destaca en su informe a nivel nacional que estas mantienen a un promedio de 13.4 asociados por empresa con una tasa de rotación del personal de alrededor del $7 \%$ (Giarelli, 2013).

De igual forma se cita que la mayoría (65\%) de las 23 empresas visitadas no se encuentran adscritas a las entidades estatales o privadas pertinentes al rubro (como la AHTI o Cámara de Industria y Comercio de Tegucigalpa), reflejando un profundo distanciamiento entre el empresariado y el Gobierno en esta actividad económica.

Las empresas evaluadas por el instrumento de esta investigación responden a una escala de medianas y pequeñas empresas, con la particularidad de que estas responden a iniciativas de negocios de tipo familiar (Belausteguigoitia, 2013).

En definitiva, la media de la experiencia corporativa con la que cuentan las empresas evaluadas en esta investigación oscila en 3 años, denotando que la incertidumbre en este sector es alta; de igual manera, se enfatiza que las 23 empresas censadas en este análisis centran sus actividades en los siguientes rubros: productos de desarrollo de software, servicios de software, productos de hardware y servicios para productos de hardware.

Los elementos citados con anterioridad responden a los 4 rubros identificados por la $\mathrm{AHTI}$, los cuales se muestran a nivel nacional en el gráfico 1. 
Gráfico 1. Campos de gestión en las empresas vinculadas a la AHTI

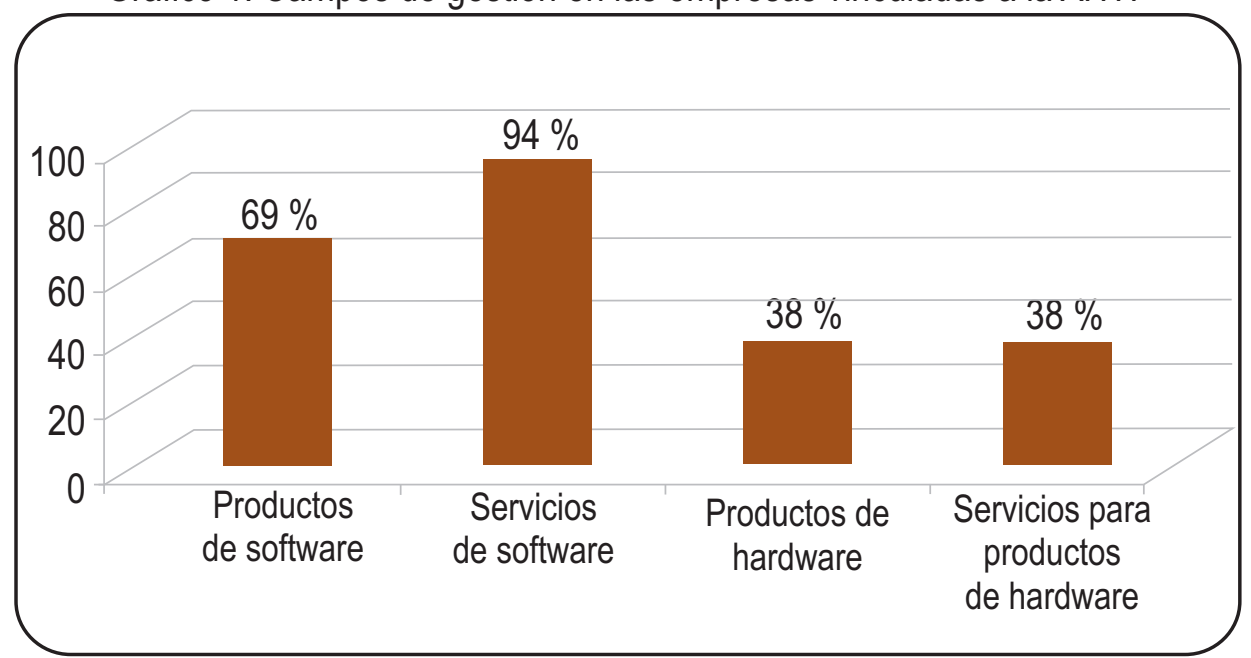

Fuente: AHTI, 2013

Perfil de los ingenieros desarrolladores de software en Honduras

Tras describir de forma holística el perfil de las empresas evaluadas, es vital para efectos de este análisis la identificación de todos los asociados pertenecientes a estas organizaciones, dado que estos corresponden de forma inmediata a la unidad de análisis en este estudio. En consecuencia y con el afán de describir los rasgos más generales de esta unidad de análisis, se puede inferir lo siguiente:

1. Edad promedio de los profesionales: 26 .

2. Nivel académico (ultima titulación obtenida): pregrado.

3. Promedio salarial devengado: entre 15,000 y 20,000 lempiras mensuales.

4. Nivel de participación por género: $70 \%$ hombres y $30 \%$ mujeres.

5. No bilingües: $50 \%$ de los encuestados.

6. Bilingües: $50 \%$ de los encuestados. 


\section{ANÁLISIS HIPÓTESIS}

1. La hipótesis 1 procura la verificación de los estándares tipificados por la AHTI, con relación a la media salarial definida, la cual se estima entre 10,000 y 15,000 lempiras (Giarelli, 2013). Tras recopilar los insumos con el instrumento de medición y posteriormente cuantificar el grado de la competitividad del capital intelectual de la unidad de análisis, se comprobó que el valor monetizado de los profesionales en la ingeniería que laboran en el sector desarrollador de software, es de 42,800 lempiras, en un intervalo de 5,000 arriba de los límites arancelarios tipificados por la AHTI (Giarelli, 2013). Por tanto, se acepta la hipótesis alternativa $(\mathrm{H} 1)$, la cual afirma que la cuantificación del capital intelectual de los profesionales en la rama de la ingeniería que laboran en el sector desarrollador de software es superior a los 30,000.00 lempiras.

2. En la hipótesis 2 se condensa el análisis de la investigación en torno a la búsqueda de la equidad entre el capital intelectual cuantificado en lempiras y la tasa salarial mensual devengada por los profesionales que laboran en el sector desarrollador de software en Tegucigalpa. En el desarrollo de esta hipótesis se hizo el análisis en el marco de 4 perspectivas:

a. Desde la óptica de la media salarial acorde con el diagnóstico del sector desarrollado por la AHTI, quienes plantean una media arancelaria entre 10,000 -15,000 lempiras (Giarelli, 2013).

b. Desde la óptica del Colegio de Ingenieros Mecánicos, Eléctricos y Químicos de Honduras y Áreas a Fin (CIMEQH), quienes tipifican que el salario de un profesional de la ingeniería de cualquier rama debería de oscilar entre 40,000 y 45,000 lempiras (CIMEQH, 1998).

c. Desde la óptica de los datos recabados por el instrumento de medición en materia de la tasa salarial, el cual describe que la unidad de análisis devenga un promedio salarial que oscila entre los 15,000 y 20,000 lempiras.

d. Desde la óptica de la cuantificación del capital intelectual, el cual tipifica que a los profesionales que laboran en el sector desarrollador de software se les debería de remunerar con un salario de 42,800 lempiras, el cual consolida las competencias requeridas para desempeñarse con suceso en el medio.

Una vez descritas las 4 perspectivas de la hipótesis, cabe articularlas para luego inferir sobre la aceptación de las hipótesis nula o alternativa, respecto a este 
planteamiento. Se destaca entre estas la variación entre la información facilitada por la AHTI y la información capturada por el informe, dado que la AHTI contempla elementos a nivel nacional que podrían alterar la media de la tasa arancelaria en estudio.

Finalmente, lo que compete es evaluar el $\mathrm{Cl}$ monetizado versus la tasa mensual devengada por los profesionales reflejados en el estudio, la cual demuestra la no existencia de un equilibrado sistema de remuneración en el sector, al restar ambos valores:

$\mathrm{Cl}$ cuantificado - tasa salarial encontrada por el estudio = subvaloración del personal

Por lo anterior, se acepta la hipótesis alternativa $(\mathrm{H} 1)$, la cual propone que la cuantificación de los profesionales en la rama de la ingeniería que laboran en el sector desarrollador de software demostró que su capital intelectual monetizado es superior (>) a su remuneración actual de mercado, lo que sugiere una subvaloración del profesional en este sector.

\section{RESULTADOS}

La tabla 3 consolida los resultados obtenidos a la luz de las hipótesis planteadas.

Tabla 3. Resumen de los resultados de la investigación

\begin{tabular}{|c|c|c|c|c|}
\hline \multicolumn{2}{|c|}{ Relación entre variables } & $\begin{array}{l}\text { Existe relación / } \\
\text { No existe relación }\end{array}$ & $\begin{array}{c}\text { Nivel de } \\
\text { significancia } \\
\% 5\end{array}$ & $\begin{array}{l}\text { Hipótesis } \\
\text { aceptada }\end{array}$ \\
\hline $\begin{array}{c}\text { Capital } \\
\text { intelectual }\end{array}$ & Tasa salarial & $\begin{array}{l}\text { No existe relación } \\
\text { entre las variables }\end{array}$ & 0.05 & $\begin{array}{c}\text { Hipótesis nula } \\
\text { (Ho) }\end{array}$ \\
\hline \multicolumn{2}{|c|}{ Valor del capital intelectual } & 42,800 lempiras & & \\
\hline \multicolumn{2}{|c|}{$\begin{array}{l}\text { Tasa salarial remunerada en } \\
\text { el mercado laboral de TGU } \\
\text { para el sector }\end{array}$} & $15,000-20,000$ lempiras & & \\
\hline \multicolumn{2}{|c|}{ Conclusión } & $\begin{array}{l}\text { Existe una subvalo. } \\
\text { ración de los profe- } \\
\text { sionales que laboran er } \\
\text { el sector desarro-llado } \\
\text { de software en TGU }\end{array}$ & & \\
\hline
\end{tabular}

Fuente: elaboración propia 
La tabla 3 condensa los resultados del estudio, entre los cuales se destaca que en el contexto hondureño y de forma particular en el sector desarrollador de software no hay evidencia estadística que permita inferir sobre la inherencia de la variable capital intelectual sobre la tasa salarial. Aseveración que es respaldada por la enorme brecha/subvaloración manifiesta entre el $\mathrm{Cl}$ cuantificado y la tasa salarial devengada (ponderada en 22,800 lempiras).

De forma iterada compete describir varios indicadores claves propios del sector desarrollador de software en la ciudad de Tegucigalpa, Francisco Morazán. Esta propuesta de investigación tiene a fin resaltar lo siguiente:

1. Los profesionales de la rama de la ingeniería que trabajan en el sector desarrollador de software en su gran mayoría son varones $(67 \%$ de predominancia), con una edad promedio de 26 años, de los cuales un $50 \%$ de ellos es bilingüe.

2. Este sector, a diferencia otros ámbitos manufactureros, de servicios, etc., presenta la particularidad de que su capacidad de absorción y adquisición de talento humano es alta, sin embargo, dadas las características de la oferta en el mercado laboral hondureño y ante la carencia de mano de obra calificada, limitan o condicionan la capacidad instalada de las empresas en el sector tecnológico, al adquirir empleados con insuficientes conocimientos, pericias 0 competencias.

3. Los profesionales que laboran en el sector desarrollador de software en su gran mayoría cuentan con una titulación a nivel de pregrado (más del $93 \%$ de los encuestados).

4. Las empresas pertenecientes al sector desarrollador de tecnología en su gran mayoría no cuentan con una certificación a nivel ISO, CMMI, IT MARK o SPINE, de hecho solamente un $19 \%$ de ellas cuenta con una certificación que les acredite como entidades procuradoras de la calidad en sus productos.

5. Los techos salariales promedio detectados por el instrumento de investigación en este análisis son los siguientes:

a. Valor mínimo (15,000.00 lempiras)

b. Valor máximo $(20,000.00$ lempiras $)$ 
c. La AHTI reveló que el techo salarial máximo en este mercado oscila en alrededor de 40,000 lempiras (USD 2,000.00).

\section{CONCLUSIONES}

A continuación se presentan las conclusiones abordadas por el análisis efectuado en esta investigación:

1. Al contrastar la tasa salarial mensualmente devengada (salario promedio mensual) con el capital intelectual monetizado de los profesionales de la rama de la ingeniería que laboran en el sector desarrollador de tecnología, se concluye que existe un desequilibrio significativo entre ambos, al manifestarse en el contexto una subvaloración del capital intelectual ponderada en alrededor de 22,800 lempiras.

2. Al evaluar la inherencia del capital intelectual $(\mathrm{Cl})$ sobre la tasa salarial mensualmente devengada (TSMD) por los profesionales de la rama de la ingeniería que laboran en el sector desarrollador de software, en la ciudad de Tegucigalpa, Francisco Morazán, se concluye que el Cl no incide sobre la tasa salarial devengada, tras observar la brecha ponderada de 22,800 lempiras, entre el $\mathrm{Cl}$ y la tasa mensual devengada, denotando una considerable subvaloración del Cl. Mostrando así una fisura en el mercado laboral del sector, la cual debería ser mitigada por el empresariado de la industria a modo de procurar la nivelación entre ambos.

\section{BIBLIOGRAFÍA}

Argueta, J. (2013). An Empirical Approach of the Impact of the Human Capital Engineering towards the Lean Manufacturing Organizational Peformance. IAMB WashingtonAnual Conference.

Argueta, J. (2013). An Empirical Approach towards the Internationalization of the Honduran Higher Education Programs. IAMB Lisbon Congres.

Argueta, J. D. (2013). An Internationalization approach: The development of a Scholarship and Study grants Observatory for Academic Performance in Central America. IAMB Anual Conference.

Becker, G. (1964). Human Capital. New York: Columbia University Press. 
Belausteguigoitia, I. (2013). Administración de empresas familiares. México: Mc Graw Hill.

Bondi, M. (2001). El capital intelectual. Los recursos humanos como recursos de las empresas. Desarrollo y Gestión. Buenos Aires: Errepar.

Campos, E. B. (1999). Experiencias de medición del capital intelectual en España. Madrid: Instituto Complutense de Madrid.

Campos, E. B. (2003). Modelo intellectus: medición y gestión del capital intelectual. Madrid: Universidad Autónoma de Madrid.

CIMEQH. (1998). Normativas de/ CIMEQH. Tegucigalpa.

Giarelli, J. (2013). Consolidado fichas informativas de las empresas afiliadas. Informe AnualAHTI. Tegucigalpa.

Pfeffer, J. (1994). Competitive Advantage through People: Unleashing the Power of the Work Force. Boston: Harvard Business School Press.

Zacapa, T. G. (2005). Diseño de un modelo para la medición del capital. Revista UNMSM, 8, 33-41. Perú 